\title{
An interior gradient estimate for the mean curvature equation of Killing graphs and applications
}

\author{
M. Dajczer, J. H. de Lira ${ }^{\dagger}$ and J. Ripoll ${ }^{\ddagger}$
}

\begin{abstract}
We extend the interior gradient estimate due to Korevaar-Simon [8] for solutions of the mean curvature equation from the case of Euclidean graphs to the general case of Killing graphs. Our main application is the proof of existence of Killing graphs with prescribed mean curvature function for continuous boundary data, thus extending a result due to Dajczer, Hinojosa and Lira [2]. In addition, we prove the existence and uniqueness of radial graphs in hyperbolic space with prescribed mean curvature function and asymptotic boundary data at infinity.
\end{abstract}

\section{Introduction}

Gradient bounds are fundamental a priori estimates for elliptic and parabolic equations and play a key role in geometry and PDE. One of the most important application of interior gradient estimates is the proof due to Serrin [12] of the solvability of the Dirichlet problem for the constant mean curvature hypersurface equation in a bounded $C^{2}$ domain of the Euclidean space for just continuous boundary data; see Theorem 16.11 of [7].

In this paper, we extend the interior gradient estimate due to Korevaar-Simon [8] (see also [9]) for solutions of the prescribed mean curvature equation in Euclidean space to the general case of Killing graphs. Our main application is the extension from $C^{2, \alpha}$ to $C^{0}$ boundary data of the result in [2] on the solvability of the Dirichlet problem for the prescribed mean curvature equation, in particular, extending Theorem 16.11 of [7] to Killing graphs. In turn, this result has an interesting application in the case of ambient spaces of constant sectional curvature, in particular, for Plateau's problem for constant mean curvature hypersurfaces inside a solid torus in Euclidean space; see our Theorem 3 below.

In addition to the above, we use our gradient estimate and a result in [2] on the Dirichlet problem on bounded domains for $C^{2, \alpha}$ boundary data to show the solvability of

*Partially supported by CNPq and FAPERJ.

${ }^{\dagger}$ Partially supported by CNPq and FUNCAP/PRONEX.

${ }^{\ddagger}$ Partially supported by CNPq. 
the asymptotic Dirichlet problem for radial graphs in the hyperbolic space with prescribed mean curvature function. A similar result for constant mean curvature was obtained in [1] using a different approach. We observe that an interior gradient estimate for Killing graphs was recently used in [10] to show the existence of nonparametric solutions for a capillary problem in warped products. Other applications of the Korevaar-Simon method can be found in [3] and [4].

To give the precise statements of our results we first discuss the geometric setting.

Let $N^{n+1}$ denote an $(n+1)$-dimensional Riemannian manifold carrying a non-singular complete Killing vector field $Y$ whose orthogonal distribution is integrable. Fix a integral hypersurface $M^{n}$ of the orthogonal distribution. Observe that $M^{n}$ is a totally geodesic submanifold of $N^{n+1}$. Let $\Omega \subset M^{n}$ be a domain such that the flow $\Psi: \mathbb{R} \times M^{n} \rightarrow N^{n+1}$ generated by $Y$ has complete orbits. Notice that $\gamma=1 /\langle Y, Y\rangle$ can be seen as a function in $\bar{\Omega}$ since $Y \gamma=0$ by the Killing equation. Moreover, the solid cylinder $\Psi(\mathbb{R} \times \bar{\Omega})$ with the induced metric has a warped product Riemannian structure $\bar{\Omega} \times{ }_{\rho} \mathbb{R}$ where $\rho=1 / \sqrt{\gamma}$.

Given a function $u$ on $\bar{\Omega}$ the associated Killing graph is the hypersurface

$$
\operatorname{Gr}(u)=\{\Psi(u(x), x): x \in \bar{\Omega}\} .
$$

It was shown in [2] that $\operatorname{Gr}(u)$ has mean curvature $H(x)$ if and only if $u \in C^{2}(\Omega)$ satisfies

$$
\mathcal{Q}[u]=\operatorname{div}\left(\frac{\nabla u}{w}\right)-\frac{\gamma}{w}\left\langle\nabla u, \bar{\nabla}_{Y} Y\right\rangle=n H
$$

where $w=\sqrt{\gamma+|\nabla u|^{2}}$ and $H$ is computed for the orientation given by the Gauss map

$$
N=\frac{1}{w}\left(\gamma Y-\Psi_{*} \nabla u\right)
$$

where $\nabla$ and div denote the gradient and divergence in $M^{n}$ and $\bar{\nabla}$ the Riemannian covariant derivative in $N^{n+1}$.

Given a point $o \in \Omega$, let $r>0$ be such that $r<i(o)$ where $i(o)$ is the injectivity radius of $M^{n}$ at $o$. We denote by $B_{r}(o)$ the geodesic ball contained in $\Omega$ centered at $o$ and radius $r$. We now state our interior gradient estimate for Killing graphs. A similar result but just for product ambient spaces $N^{n+1}=M^{n} \times \mathbb{R}$ was given in [13].

Theorem 1. Let $u \in C^{3}\left(B_{r}(o)\right)$ be a negative solution of the mean curvature equation (11) for $H \in C^{1}(\Omega)$. Then, there exists a constant $L=L(u(o), r, \gamma, H)$ such that $|\nabla u(o)| \leq L$.

The Killing cylinder $K$ over $\Gamma=\partial \Omega$ is the hypersurface in $N^{n+1}$ ruled by the flow lines of $Y$ through $\Gamma$ given by

$$
K=\{q=\Psi(s, x): s \in \mathbb{R} \text { and } x \in \Gamma\} .
$$

In the sequel, the mean curvature of $K$ pointing inward is denoted by $H_{\text {cyl }}$.

The use of Theorem 1 gives the following extension of the main result in [2]. 
Theorem 2. Let $\Omega \subset M^{n}$ be a $C^{2, \alpha}$ bounded domain satisfying $H_{c y l} \geq 0$ and

$$
\inf _{\Omega} \underset{M}{\operatorname{Ric}} \geq-n \inf _{\Gamma} H_{c y l}^{2} .
$$

Let $H \in C^{\beta}(\Omega)$ for some $0<\beta<1$ be such that $\sup _{\Omega}|H| \leq \inf _{\Gamma} H_{\text {cyl }}$. Given $\varphi \in C^{0}(\Gamma)$ there exists a unique function $u \in C^{2, \beta}(\Omega) \cap C^{0}(\bar{\Omega})$ whose Killing graph has mean curvature $H$ and $\left.u\right|_{\Gamma}=\varphi$.

Similar extensions of Theorems 2 and 3 of [2] (proved under weaker assumptions on the Ricci curvature of $M^{n}$ than in Theorem 1 in that paper) also follow. Observe also that Theorem 2 generalizes Theorem 1.4 of [13].

Although our setting is very general and the proof is based on nonparametric methods, it turns out that our Theorem 2 has an interesting consequence on Plateau's problem for constant mean curvature hypersurfaces in $\mathbb{R}^{n+1}$, the hyperbolic space $\mathbb{H}^{n+1}$ and the sphere $\mathbb{S}^{n+1}$. Let $\mathbb{Q}^{n+1}$ stand for any of these spaces and let $\mathbb{Q}_{+}^{n}$ be a half totally geodesic hypersurface of $\mathbb{Q}^{n+1}$, hence $\mathbb{Q}_{+}^{n}$ is complete up to the boundary and $\partial \mathbb{Q}_{+}^{n}$ is a totally geodesic submanifold of $\mathbb{Q}^{n+1}$ of codimension two. We denote by $T$ the solid torus in $\mathbb{Q}^{n+1}, n \geq 2$, obtained by rotating a $C^{2, \alpha}$ domain $\Omega \subset \mathbb{Q}_{+}^{n} \backslash \partial \mathbb{Q}_{+}^{n}$ diffeomorphic to a ball around $\partial \mathbb{Q}_{+}^{n}$ and by $H_{\mathcal{T}}$ the mean curvature of its boundary $\mathcal{T}$ with respect to the inner orientation.

Theorem 3. Assume that $\Omega$ is $C^{2, \alpha}$ and that $H_{\mathcal{T}} \geq H$ for some constant $H \geq 0$. If $\Gamma \subset \mathcal{T}$ is a compact embedded topological hypersurface of $\mathcal{T}$ that intersects at a single point every circle through $\partial \Omega$ orthogonal to $\mathbb{Q}_{+}^{n}$, then there exists a unique compact embedded topological hypersurface $M^{n} \subset T$ with boundary $\Gamma$ homeomorphic to a closed ball that is $C^{\infty}$ and has constant mean curvature $H$ on $M^{n} \backslash \Gamma$.

Observe that the mean curvature with respect to the inner orientation of a geodesic sphere in $\mathbb{Q}_{+}^{n} \backslash \partial \mathbb{Q}_{+}^{n}$ with radius $r$ tends to $+\infty$ as $r \rightarrow 0$. Thus, the mean curvature of the boundary of the solid torus obtained by rotating the geodesic ball in $\mathbb{Q}_{+}^{n} \backslash \partial \mathbb{Q}_{+}^{n}$ has mean curvature larger than any given $H>0$ if the radius of the ball is small enough, hence Theorem 3 applies to such a torus.

In this paper, the other application of Theorem 1 is to prove the solvability of the asymptotic Dirichlet problem for radial graphs in the hyperbolic space with prescribed mean curvature function. Such a result but for constant mean curvature was obtained in [1] by a different approach. Our solution to the problem is obtained as the limit of radial graphs with prescribed mean curvature on a sequence of concentric geodesic balls exhausting $\mathbb{H}^{n}$. The existence of these Killing graphs on geodesic balls follows from the general result in [2].

Let $\mathbb{H}^{n}$ be a complete totally geodesic hypersurface in $\mathbb{H}^{n+1}$. A fixed constant speed geodesic line $\ell$ orthogonal to $\mathbb{H}^{n}$ at a point $o \in \mathbb{H}^{n}$ determines uniquely a one-parameter 
family of translation isometries in $\mathbb{H}^{n+1}$ that preserve $\ell$ and whose Killing field $Y$ extends the velocity vector of $\ell$. These isometries extend to the ideal boundary at infinity $\partial_{\infty} \mathbb{H}^{n+1}$ of $\mathbb{H}^{n+1}$ as conformal transformations belonging to the conformal structure of $\partial_{\infty} \mathbb{H}^{n+1}$.

Denote by $\Psi: \mathbb{R} \times \mathbb{H}^{n} \rightarrow \mathbb{H}^{n+1}$ the flux generated by $Y$ and set $\overline{\mathbb{H}}^{n}=\mathbb{H}^{n} \cup \partial_{\infty} \mathbb{H}^{n}$. Given a function $u \in C^{2}\left(\mathbb{H}^{n}\right) \cap C^{0}\left(\overline{\mathbb{H}}^{n}\right)$ its radial graph (Killing graph) is the hypersurface

$$
\operatorname{Gr}(u)=\left\{\Psi(u(x), x): x \in \mathbb{H}^{n}\right\}
$$

with asymptotic boundary given by

$$
\partial_{\infty} \operatorname{Gr}(u)=\overline{\operatorname{Gr}(u)} \cap \partial_{\infty} \mathbb{H}^{n+1} .
$$

With some abuse of language, we say that $\operatorname{Gr}(\phi)=\left\{\Psi(\phi(x), x): x \in \partial_{\infty} \mathbb{H}^{n}\right\}$ is the radial graph of the function $\phi=\left.u\right|_{\partial_{\infty} \mathbb{H}^{n}}$.

Theorem 4. Given $H \in C^{\beta}\left(\mathbb{H}^{n}\right), 0<\beta<1$, with $\sup _{\mathbb{H}^{n}}|H|<1$ and $\phi \in C^{0}\left(\partial_{\infty} \mathbb{H}^{n}\right)$ there exists a unique function $u \in C^{2, \beta}\left(\mathbb{H}^{n}\right) \cap C^{0}\left(\overline{\mathbb{H}}^{n}\right)$ such that $G r(u)$ has mean curvature $H$ and $\partial_{\infty} G r(u)$ is the radial graph of $\phi$.

The above result is a natural extension to Killing graphs in the hyperbolic space of the asymptotic Dirichlet problem for the mean curvature hypersurface PDE, which in the minimal case has been studied in a complete noncompact Riemannian manifold in [5], 6] and [11].

\section{The gradient estimate}

For a system of coordinates $x=\left(x^{1}, \ldots, x^{n}\right)$ equation (11) reads as

$$
a^{i j} u_{i ; j}-R\langle\nabla \gamma, \nabla u\rangle=n H w \quad \text { where } \quad a^{i j}=\sigma^{i j}-\frac{u^{i} u^{j}}{w^{2}} .
$$

Here the $\sigma_{i j}$ 's are the coefficients of the metric in $M^{n}$ and for simplicity we denote

$$
R=\frac{\gamma+w^{2}}{2 \gamma w^{2}}
$$

Proof of Theorem 1: We define a nonnegative function $\eta(x)=g(\phi(x))$ on $B_{r}(o)$ where

$$
g(t)=e^{C_{1} t}-1
$$

for some constant $C_{1}>0$ and $\phi$ is given by

$$
\phi(x)=\left(1-\frac{d^{2}(x)}{r^{2}}+\frac{u(x)}{2 u_{0}}\right)^{+} .
$$


Here + means positive part, $d(x)$ is the geodesic distance to $o$ in $M^{n}$ and $C=1 / 2 u_{o}$ where $u_{0}=-u(o)$. Then $\eta$ vanishes outside of $B_{r}(o)$. To be more precise, we should replace 1 by $1-\epsilon$ in the definition of $\phi$ so that $\eta$ is smooth with compact support and later let $\epsilon$ tend to zero, but this is omitted for simplicity.

Let $p \in B_{r}(o)$ be an interior point where the function $h=\eta w$ has a maximum. In the sequel computations are done at this point without further notice. From $h_{i}=0$ we have

$$
\eta_{i} w=-\eta w_{i}
$$

Moreover, the Hessian matrix of $h$ is negative semidefinite. Taking the trace of the product of the Hessian matrix of $h$ with the positive definite matrix $a^{i j} / w$ yields

$$
0 \geq \frac{1}{w} a^{i j} h_{i ; j}=\frac{a^{i j}}{w}\left(w \eta_{i ; j}+2 \eta_{i} w_{j}+\eta w_{i ; j}\right)
$$

We obtain using (4) that

$$
a^{i j} \eta_{i ; j}+\frac{\eta}{w^{2}} a^{i j}\left(w w_{i ; j}-2 w_{i} w_{j}\right) \leq 0
$$

From (2) we have

$$
N^{k}=-\frac{u^{k}}{w}
$$

Thus,

$$
w_{i}=\frac{\gamma_{i}}{2 w}+\frac{u^{k} u_{k ; i}}{w}=\frac{\gamma_{i}}{2 w}-N^{k} u_{k ; i}
$$

In the sequel, we use (44), (6) and (7) several times without further reference.

We have,

$$
\begin{aligned}
w_{i ; j} & =\frac{\gamma_{i ; j}}{2 w}-\frac{\gamma_{i} w_{j}}{2 w^{2}}-N_{; j}^{k} u_{k ; i}-N^{k} u_{k ; i j} \\
& =\frac{\gamma_{i ; j}}{2 w}+\frac{\sigma^{k l}}{w} u_{l ; j} u_{k ; i}-\frac{w}{\eta^{2}} \eta_{i} \eta_{j}-N^{k} u_{k ; i j} \\
& =\frac{\gamma_{i ; j}}{2 w}+\frac{1}{w}\left(\sigma^{k l}-N^{k} N^{l}\right) u_{l ; j} u_{k ; i}+\frac{1}{w} N^{k} N^{l} u_{l ; j} u_{k ; i}-\frac{1}{w} w_{i} w_{j}-N^{k} u_{k ; i j} \\
& =\frac{\gamma_{i ; j}}{2 w}+\frac{a^{k l}}{w} u_{l ; j} u_{k ; i}+\frac{\gamma_{i} \gamma_{j}}{4 w^{3}}-\frac{1}{2 w^{2}}\left(w_{i} \gamma_{j}+w_{j} \gamma_{i}\right)-N^{k} u_{k ; i j} .
\end{aligned}
$$

Thus,

$$
a^{i j} w_{i ; j}=\frac{1}{2 w} a^{i j} \gamma_{i ; j}+\frac{1}{w} a^{i j} a^{k l} u_{l ; j} u_{k ; i}+\frac{1}{4 w^{3}} a^{i j} \gamma_{i} \gamma_{j}+\frac{1}{w \eta} a^{i j} \eta_{i} \gamma_{j}-N^{k} a^{i j} u_{k ; i j}
$$

We compute the last term of (8)). The Ricci identities for the Hessian of $u$ yield

$$
u_{k ; i j}=u_{i ; k j}=u_{i ; j k}+R_{k j i}^{l} u_{l} .
$$


Hence,

$$
N^{k} a^{i j} u_{k ; i j}=N^{k} a^{i j} u_{i ; j k}-\frac{1}{w} a^{i j} R_{k j i}^{l} u^{k} u_{l}=N^{k}\left(a^{i j} u_{i ; j}\right)_{; k}-N^{k} a_{; k}^{i j} u_{i ; j}-\frac{1}{w} a^{i j} R_{k j i}^{l} u^{k} u_{l} .
$$

It follows that

$$
N^{k} a^{i j} u_{k ; i j}=n N^{k}(w H)_{k}+N^{k}(R\langle\nabla \gamma, \nabla u\rangle)_{k}-N^{k} a_{; k}^{i j} u_{i ; j}-\frac{1}{w} a^{i j} R_{k j i}^{l} u^{k} u_{l} .
$$

We compute the first three terms of (9). For the first term, we have

$$
(w H)_{k}=\frac{w}{\eta}\left(\eta H_{k}-H \eta_{k}\right) .
$$

Since

$$
\left(\frac{\gamma+w^{2}}{w^{2}}\right)_{k}=\frac{\gamma_{k}}{w^{2}}-\frac{\gamma}{w^{4}}\left(\gamma_{k}+2 u^{l} u_{l ; k}\right)=\frac{1}{w^{2}}\left(\gamma_{k}+\frac{2 \gamma \eta_{k}}{\eta}\right)
$$

and

$$
\left(\frac{1}{2 \gamma}\langle\nabla \gamma, \nabla u\rangle\right)_{k}=\left(\frac{\gamma_{l}}{2 \gamma}\right)_{; k} u^{l}+\frac{\gamma^{l}}{2 \gamma} u_{l ; k}=\frac{1}{2 \gamma}\left[\left(\frac{\gamma_{l} \gamma_{k}}{\gamma}-\gamma_{k ; l}\right) w N^{l}+\gamma^{l} u_{l ; k}\right]
$$

we obtain for the second term of (9) that

$$
(R\langle\nabla \gamma, \nabla u\rangle)_{k}=R\left[\left(\frac{\gamma_{l} \gamma_{k}}{\gamma}-\gamma_{k ; l}\right) w N^{l}+\gamma^{l} u_{l ; k}\right]+\frac{1}{w^{2}}\left(\frac{\gamma_{k}}{2 \gamma}+\frac{\eta_{k}}{\eta}\right)\langle\nabla \gamma, \nabla u\rangle .
$$

We have,

$$
\begin{aligned}
a_{; k}^{i j} & =-\frac{1}{w^{2}}\left(u_{; k}^{i} u^{j}+u^{i} u_{; k}^{j}\right)+\frac{1}{w^{4}}\left(\gamma_{k}-2 w N^{l} u_{l ; k}\right) u^{i} u^{j} \\
& =\frac{1}{w}\left(u_{; k}^{i}-N^{i} N^{l} u_{l ; k}\right) N^{j}+\frac{1}{w}\left(u_{; k}^{j}-N^{j} N^{l} u_{l ; k}\right) N^{i}+\frac{1}{w^{2}} \gamma_{k} N^{i} N^{j} \\
& =\frac{1}{w} a^{i l} u_{l ; k} N^{j}+\frac{1}{w} a^{j l} u_{l ; k} N^{i}+\frac{1}{w^{2}} \gamma_{k} N^{i} N^{j} .
\end{aligned}
$$

It follows that the third term of (9) is given by

$$
N^{k} a_{; k}^{i j} u_{i ; j}=\frac{2}{w} a^{i j}\left(\frac{w \eta_{i}}{\eta}+\frac{\gamma_{i}}{2 w}\right)\left(\frac{w \eta_{j}}{\eta}+\frac{\gamma_{j}}{2 w}\right)+\frac{1}{w^{2}} \gamma_{k} N^{k} N^{i}\left(\frac{w \eta_{i}}{\eta}+\frac{\gamma_{i}}{2 w}\right) .
$$

Replacing (10), (11) and (12) into (9) ) yields

$$
\begin{aligned}
N^{k} a^{i j} u_{k ; i j} & =n \frac{w}{\eta} N^{k}\left(\eta H_{k}-H \eta_{k}\right)-\frac{2}{w} a^{i j}\left(\frac{w \eta_{i}}{\eta}+\frac{\gamma_{i}}{2 w}\right)\left(\frac{w \eta_{j}}{\eta}+\frac{\gamma_{j}}{2 w}\right) \\
& -\frac{1}{w^{2}} \gamma_{k} N^{k} N^{i}\left(\frac{w \eta_{i}}{\eta}+\frac{\gamma_{i}}{2 w}\right)+\frac{1}{w^{2}} N^{k}\left(\frac{\gamma_{k}}{2 \gamma}+\frac{\eta_{k}}{\eta}\right)\langle\nabla \gamma, \nabla u\rangle \\
& +R\left[\left(\frac{\gamma}{2 w} \sigma^{k l}+w N^{k} N^{l}\right) \frac{\gamma_{k} \gamma_{l}}{\gamma}-w N^{k} N^{l} \gamma_{k ; l}+\frac{w}{\eta} \gamma_{l} \eta^{l}\right]-\frac{1}{w} a^{i j} R_{k j i}^{l} u^{k} u_{l} .
\end{aligned}
$$


It now follows from (8) that

$$
\begin{aligned}
& a^{i j} w_{i ; j}-\frac{2}{w} a^{i j} w_{i} w_{j}=\frac{3}{4 w^{3}} a^{i j} \gamma_{i} \gamma_{j}+\frac{1}{w} a^{i j} a^{k l} u_{l ; j} u_{k ; i}+\frac{3}{w \eta} a^{i j} \gamma_{i} \eta_{j}+\frac{1}{2 w} a^{i j} \gamma_{i ; j} \\
& +\frac{1}{w} a^{i j} R_{k j i}^{l} u^{k} u_{l}-n N^{k} \frac{w}{\eta}\left(\eta H_{k}-H \eta_{k}\right)+\frac{1}{w^{2}} \gamma_{k} N^{k} N^{i}\left(\frac{w \eta_{i}}{\eta}+\frac{\gamma_{i}}{2 w}\right) \\
& -\frac{1}{w^{2}} N^{k}\left(\frac{\gamma_{k}}{2 \gamma}+\frac{\eta_{k}}{\eta}\right)\langle\nabla \gamma, \nabla u\rangle-R\left[\left(\frac{\gamma}{2 w} \sigma^{k l}+w N^{k} N^{l}\right) \frac{\gamma_{k} \gamma_{l}}{\gamma}-w N^{k} N^{l} \gamma_{k ; l}+\frac{w}{\eta} \gamma_{l} \eta^{l}\right] .
\end{aligned}
$$

After multiplying both sides by $\eta / w$ and discarding the non-negative terms, we obtain

$$
\begin{aligned}
& \frac{\eta}{w}\left(a^{i j} w_{i ; j}-\frac{2}{w} a^{i j} w_{i} w_{j}\right) \geq\left[-n N^{k} H_{k}-\frac{\gamma_{k}}{2 \gamma w^{3}} N^{k}\langle\nabla \gamma, \nabla u\rangle+\frac{1}{2 w^{2}} a^{i j} \gamma_{i ; j}\right. \\
& \left.-R\left(\left(\frac{\gamma}{2 w^{2}} \sigma^{k l}+N^{k} N^{l}\right) \frac{\gamma_{k} \gamma_{l}}{\gamma}-N^{k} N^{l} \gamma_{k ; l}\right)+\frac{1}{w^{2}} a^{i j} R_{k j i}^{l} u^{k} u_{l}\right] \eta \\
& +\left[\left(n H+\frac{1}{w^{2}} N^{k} \gamma_{k}-\frac{1}{w^{3}}\langle\nabla \gamma, \nabla u\rangle\right) N^{i}+\left(\frac{3}{w^{2}} a^{i j}-R \sigma^{i j}\right) \gamma_{j}\right] \eta_{i} .
\end{aligned}
$$

It is easy to check that there is a positive constant $M=M(\gamma, H)$ such that

$$
\frac{1}{w^{2}} \eta a^{i j}\left(w w_{i ; j}-2 w_{i} w_{j}\right) \geq-M \eta-A^{i} \eta_{i}
$$

where $-A^{i}$ denotes the coefficient of $\eta_{i}$. It follows from (15) and (13) that

$$
a^{i j} \eta_{i ; j}-M \eta-A^{i} \eta_{i} \leq 0
$$

On the other hand,

$$
\left.\eta_{i}=g^{\prime-2}\left(d^{2}\right)_{i}+C u_{i}\right)
$$

and

$$
\left.\left.\eta_{i ; j}=g^{\prime-2}\left(d^{2}\right)_{i ; j}+C u_{i ; j}\right)+g^{\prime \prime-2}\left(d^{2}\right)_{i}+C u_{i}\right)\left(-r^{-2}\left(d^{2}\right)_{j}+C u_{j}\right) .
$$

Hence,

$$
\begin{aligned}
a^{i j}\left(r^{-2}\left(d^{2}\right)_{i}\right. & \left.-C u_{i}\right)\left(r^{-2}\left(d^{2}\right)_{j}-C u_{j}\right) \\
& =\frac{C^{2} \gamma}{w^{2}}|\nabla u|^{2}-\frac{2 C \gamma}{r^{2} w^{2}}\left\langle\nabla u, \nabla d^{2}\right\rangle+\frac{1}{r^{4}}\left(\left|\nabla d^{2}\right|^{2}-\frac{1}{w^{2}}\left\langle\nabla u, \nabla d^{2}\right\rangle^{2}\right) \\
& \geq \frac{C^{2} \gamma}{w^{2}}\left(|\nabla u|^{2}-\frac{2}{C r^{2}}\left\langle\nabla u, \nabla d^{2}\right\rangle\right)
\end{aligned}
$$

and

$$
a^{i j}\left(-r^{-2}\left(d^{2}\right)_{i ; j}+C u_{i ; j}\right)=-r^{-2} a^{i j}\left(d^{2}\right)_{i ; j}+C(n H w+R\langle\nabla \gamma, \nabla u\rangle)
$$

where

$$
a^{i j}\left(d^{2}\right)_{i ; j}=\Delta d^{2}-\frac{1}{w^{2}}\left\langle\nabla_{\nabla u} \nabla d^{2}, \nabla u\right\rangle
$$


It follows from (14) that

$$
\begin{aligned}
& \frac{C^{2} \gamma}{w^{2}}\left[|\nabla u|^{2}-\frac{2}{C r^{2}}\left\langle\nabla u, \nabla d^{2}\right\rangle\right] g^{\prime \prime}+\left[\frac{1}{r^{2} w^{2}}\left\langle\nabla_{\nabla u} \nabla d^{2}, \nabla u\right\rangle-\frac{\Delta d^{2}}{r^{2}}+C(n H w+R\langle\nabla \gamma, \nabla u\rangle)\right] g^{\prime} \\
& \leq M g+A^{i}\left(-r^{-2}\left(d^{2}\right)_{i}+C u_{i}\right) g^{\prime} .
\end{aligned}
$$

Since

$$
-A^{i}\left(d^{2}\right)_{i}=\left(n H+\frac{1}{w^{2}} N^{k} \gamma_{k}-\frac{1}{w^{3}}\langle\nabla \gamma, \nabla u\rangle\right) N^{i}\left(d^{2}\right)_{i}+\left(\frac{3}{w^{2}} a^{i j}-R \sigma^{i j}\right) \gamma_{j}\left(d^{2}\right)_{i}
$$

and

$$
A^{i} u_{i}=\left(n H w+\frac{1}{w} N^{k} \gamma_{k}-\frac{1}{w^{2}}\langle\nabla \gamma, \nabla u\rangle\right) \frac{|\nabla u|^{2}}{w^{2}}+\frac{3}{w} a^{i j} \gamma_{j} N_{i}+R\langle\nabla \gamma, \nabla u\rangle
$$

we conclude that

$$
\frac{C^{2} \gamma}{\gamma+|\nabla u|^{2}}\left(|\nabla u|^{2}-\frac{2}{C r^{2}}\left\langle\nabla u, \nabla d^{2}\right\rangle\right) g^{\prime \prime}+P g^{\prime}-M g \leq 0
$$

where the coefficient

$$
\begin{gathered}
P=\frac{n}{w} C H \gamma-\frac{1}{r^{2}}\left(\Delta d^{2}-\frac{1}{w^{2}}\left\langle\nabla_{\nabla u} \nabla d^{2}, \nabla u\right\rangle\right)-C\left(w N^{k} \gamma_{k}-\langle\nabla \gamma, \nabla u\rangle\right) \frac{|\nabla u|^{2}}{w^{4}} \\
-\frac{3}{w} C a^{i j} \gamma_{j} N_{i}-\frac{1}{r^{2} w^{3}}\left(n H w^{3}+w N^{k} \gamma_{k}-\langle\nabla \gamma, \nabla u\rangle\right) N^{i}\left(d^{2}\right)_{i}-\frac{1}{r^{2}}\left(\frac{3}{w} a^{i j}-R \sigma^{i j}\right) \gamma_{i}\left(d^{2}\right)_{j}
\end{gathered}
$$

of $g^{\prime}$ is bounded above by a positive constant $C_{0}=C_{0}(u(o), r, \gamma, H)$.

Suppose that

$$
|\nabla u| \geq \frac{8}{C r}=\frac{16 u_{0}}{r}
$$

Then, we have

$$
|\nabla u| \geq \frac{4\left|\nabla d^{2}\right|}{C r^{2}}
$$

and

$$
|\nabla u|^{2}-\frac{2}{C r^{2}}\left\langle\nabla u, \nabla d^{2}\right\rangle \geq|\nabla u|^{2}-\frac{2}{C r^{2}}|\nabla u|\left|\nabla d^{2}\right| \geq \frac{1}{2}|\nabla u|^{2} .
$$

Thus, there exists a constant $D=D(u(0), r, \gamma)$ such that

$$
\frac{C^{2} \gamma}{\gamma+|\nabla u|^{2}}\left(|\nabla u|^{2}-\frac{2}{C r^{2}}\left\langle\nabla u, \nabla d^{2}\right\rangle\right) \geq \frac{C^{2} \gamma|\nabla u|^{2}}{2\left(\gamma+|\nabla u|^{2}\right)} \geq D>0 .
$$

For instance, setting $\gamma_{0}=\inf _{B_{r}(o)} \gamma$ we may take $D=32 \gamma_{0} /\left(r^{2} \gamma_{0}+256 u^{2}(0)\right)$. Since $g(t)=e^{C_{1} t}-1$, we obtain a contradiction taking $C_{1}=C_{1}(u(o), r, \gamma, H)$ sufficiently large, that is, such that

$$
D g^{\prime \prime}(p)+C_{0} g^{\prime}(p)-M g(p)>0 \text {. }
$$


Hence,

$$
w(p) \leq C_{2}=\gamma_{1}+\frac{16 u_{0}}{r}
$$

where $\gamma_{1}=\sup _{B_{r}(o)} \gamma$. Since $h(o) \leq h(p)$, we obtain $\eta(o) w(o) \leq \eta(p) w(p)$. Therefore,

$$
\left(e^{C_{1} / 2}-1\right) w(o) \leq C_{2} e^{C_{1}} .
$$

This gives the desired estimate and concludes the proof.

Remark 5. Observe that the constant $L$ given by Theorem 1 also depends on the geometry of $M^{n}$ along $B_{r}(o)$ including the Ricci curvature.

\section{The applications}

In this section, we provide the proofs of the applications of Theorem 1 that has been stated in the introduction.

Proof of Theorem 2: Let $\varphi_{k}^{ \pm} \in C^{2, \alpha}(\Gamma)$ be monotonic sequences converging from above and below to $\varphi$ in the $C^{0}$ norm. Let $H_{k} \in C^{\infty}(\Omega)$ be a sequence converging to $H$ such that $H_{0}=\sup _{k} \sup _{\Omega}\left|H_{k}\right| \leq \inf _{\Gamma} H_{c y l}$. Set $H^{+}=H_{0}$ and $H^{-}=-H_{0}$. By Theorem 1 of [2] there exist solutions $u_{k}^{ \pm} \in C^{2, \alpha}(\bar{\Omega})$ of $\mathcal{Q}_{H_{k}}[u]=n H_{k}$ and $v_{k}^{ \pm} \in C^{2, \alpha}(\bar{\Omega})$ of $\mathcal{Q}_{H^{ \pm}}[v]=n H^{ \pm}$such that $\left.u_{k}^{ \pm}\right|_{\Gamma}=\varphi_{k}^{ \pm}$and $\left.v_{k}^{ \pm}\right|_{\Gamma}=\varphi_{k}^{ \pm}$. ¿From Theorem 6.17 of [7] it follows that $u_{k}^{ \pm}, v_{k}^{ \pm} \in C^{\infty}(\Omega)$. The sequences $u_{k}^{ \pm}, v_{k}^{ \pm}$have uniformly bounded $C^{0}$ norm since, by the comparison principle,

$$
v_{1}^{-} \leq \ldots \leq v_{k}^{-} \leq v_{k+1}^{-} \leq \ldots \leq v_{k+1}^{+} \leq v_{k}^{+} \leq \ldots \leq v_{1}^{+}, \quad k=1,2, \ldots
$$

and

$$
v_{k}^{-} \leq u_{k}^{ \pm} \leq v_{k}^{+}, \quad k=1,2, \ldots
$$

It follows from Theorem 1 and linear elliptic PDE theory that the sequences $u_{k}^{ \pm}, v_{k}^{ \pm}$have equicontinous $C^{2, \beta}$ norm on compact subsets of $\Omega$, the uniform $C^{2, \beta}$ norm depending only on the distance of the compact subset to $\Gamma$ (Corollary 6.3 of [7]). Considering an exhaustion of $\Omega$ by relatively compact subdomains and using the diagonal method we obtain that $u_{k}^{ \pm}$and $v_{k}^{ \pm}$contain subsequences converging uniformly on compact subsets of $\Omega$ on the $C^{2}$ norm to $u, v^{ \pm} \in C^{2}(\Omega)$ satisfying $\mathcal{Q}_{H}[u]=n H$ and $\mathcal{Q}_{H^{ \pm}}\left[v^{ \pm}\right]=n H^{ \pm}$. By (16) we have that $v^{-} \leq u \leq v^{+}$. Since $\left.v_{k}^{ \pm}\right|_{\partial \Omega}=\varphi_{k}^{ \pm}$converges to $\varphi$, it follows from (15) that $v^{ \pm}$extends continuously to $\bar{\Omega}$ and $\left.v^{ \pm}\right|_{\partial \Omega}=\varphi$. Therefore, $u$ extends continuously to $\bar{\Omega}$ and $\left.u\right|_{\partial \Omega}=\varphi$. Since $H \in C^{\beta}(\Omega)$ and $\mathcal{Q}_{H}[u]=n H$ then $u \in C^{2, \beta}(\Omega) \cap C^{0}(\bar{\Omega})$ from Theorem 6.17 of [7].

Proof of Theorem 3: The circles orthogonal to $\mathbb{Q}_{+}^{n}$ are the orbits of a Killing field orthogonal to $\mathbb{Q}_{+}^{n}$. By assumption $\Gamma$ is the graph of a continuous function $\phi \in C^{\infty}(\partial \Omega)$. It is clear that the other conditions of Theorem 2 are satisfied when applied to the universal cover of $T$, and thus the proof follows. 
Proof of Theorem 局: The proof will be done in two steps. First, we show the existence of a uniform height estimate for the solutions of the Dirichlet problem on a sequence of concentric geodesic balls exhausting $\mathbb{H}^{n}$. Then, we prove that a subsequence of these solutions converges to a solution of our problem. We may assume that $H \in C^{\infty}\left(\mathbb{H}^{n}\right)$ since the case $H \in C^{\beta}\left(\mathbb{H}^{n}\right)$ can be obtained by using approximating arguments as in the proof of Theorem 2 ,

We represent the hyperbolic space $\mathbb{H}^{n+1}$ of constant sectional curvature -1 as the warped product manifold

$$
\mathbb{H}^{n+1}=\mathbb{H}^{n} \times_{\cosh \rho} \mathbb{R}
$$

where $\rho$ is the geodesic distance in $\mathbb{H}^{n}$ to the point $o \in \mathbb{H}^{n}$ determined by the geodesic $\ell$. In terms of the notation fixed earlier $Y=\partial / \partial s$ where $s$ parametrizes the factor $\mathbb{R}$. In (1) we thus have

$$
\gamma=1 / \cosh ^{2} \rho
$$

Let $\Omega_{k}$ denote the geodesic disc in $\mathbb{H}^{n}$ of radius $\rho_{k}$ centered at $o$ with boundary $\Gamma_{k}$ where

$$
\rho_{k}=\operatorname{arctanh}(1-1 / k), \quad k=2,3, \ldots
$$

Let $F \in C^{2, \alpha}\left(\mathbb{H}^{n}\right) \cap C^{0}\left(\overline{\mathbb{H}}^{n}\right)$ be such that $\left.F\right|_{\partial_{\infty} \mathbb{H}^{n}}=\phi$ and set $M_{0}=|F|_{0}$. We claim that the unique solution $u_{k} \in C^{2}\left(\bar{\Omega}_{k}\right)$ for any $k \geq 2$ obtained in [2] of the auxiliary Dirichlet problem

$$
\left\{\begin{array}{l}
\mathcal{Q}\left[u_{k}\right]=\left.n H\right|_{\Omega_{k}} \\
\left.u_{k}\right|_{\Gamma_{k}}=\left.F\right|_{\Gamma_{k}},
\end{array}\right.
$$

has a uniform height estimate, that is, independent of $k$.

The geodesic distance $d$ from $\Gamma_{k}$ measured along normal geodesics pointing towards $o$ is $d=\rho_{k}-\rho$. The geodesic curvature of the flow lines of $Y$ is given by

$$
\kappa=\left\langle\nabla d, \bar{\nabla}_{\sqrt{\gamma} Y} \sqrt{\gamma} Y\right\rangle=-\gamma\left\langle Y, \bar{\nabla}_{\nabla d} Y\right\rangle=\frac{\gamma^{\prime}}{2 \gamma}=\tanh \rho
$$

where ' denotes derivative with respect to $d$ and we used (17). Since the mean curvature of the geodesic ball $\Gamma_{k}$ is $\operatorname{coth} \rho_{k}$, then the constant mean curvature $H_{k}$ of the Killing cylinder over $\Gamma_{k}$ is

$$
H_{k}=\frac{1}{n}\left((n-1) \operatorname{coth} \rho_{k}+\tanh \rho_{k}\right)>1
$$

with respect to the pointing inward orientation.

Consider on $\Omega_{k}$ the function $v_{k}(x)=M_{0}+h(d(x))$ where $h$ has to be chosen. Then,

$$
\begin{aligned}
\mathcal{Q}\left[v_{k}\right] & =\operatorname{div}\left(\frac{h^{\prime} \nabla d}{\sqrt{\gamma+h^{\prime 2}}}\right)-\frac{\gamma h^{\prime}}{\sqrt{\gamma+h^{\prime 2}}}\left\langle\nabla d, \bar{\nabla}_{Y} Y\right\rangle \\
& =\frac{h^{\prime}}{\sqrt{\gamma+h^{\prime 2}}}(\Delta d-\kappa)+\left(\frac{h^{\prime}}{\sqrt{\gamma+h^{\prime 2}}}\right)^{\prime} .
\end{aligned}
$$


Since $-\frac{1}{n-1} \Delta d$ is the mean curvature of the geodesic sphere in $\mathbb{H}^{n}$ of radius $\rho=\rho_{k}-d$, we have that

$$
\Delta d-\kappa=-n H_{k}
$$

Thus,

$$
\mathcal{Q}\left[v_{k}\right]=-\frac{n h^{\prime} H_{k}}{\sqrt{\gamma+h^{\prime 2}}}+\frac{\gamma\left(h^{\prime \prime}-\kappa h^{\prime}\right)}{\left(\gamma+h^{\prime 2}\right)^{\frac{3}{2}}} .
$$

We choose

$$
h(d)=C\left(\arcsin \left(\tanh \rho_{k}\right)-\arcsin (\tanh \rho)\right)
$$

where $C>0$ is a constant to be chosen and arcsin : $[-1,1] \rightarrow\left[-\frac{\pi}{2}, \frac{\pi}{2}\right]$. Thus

$$
h^{\prime}=C / \cosh \rho \text { and } \quad h^{\prime \prime}=C \tanh \rho / \cosh \rho .
$$

It follows from (18) that $h^{\prime \prime}-\kappa h^{\prime}=0$ and using (17) that

$$
\frac{h^{\prime}}{\sqrt{\gamma+h^{\prime 2}}}=\frac{C}{\sqrt{1+C^{2}}} \text {. }
$$

Therefore,

$$
\mathcal{Q}\left[v_{k}\right]=-\frac{C}{\sqrt{1+C^{2}}} n H_{k} .
$$

Hence, in order to obtain that $Q\left[v_{k}\right]<Q\left[u_{k}\right]=n H$ it suffices to choose $C$ such that

$$
\frac{C}{\sqrt{1+C^{2}}} H_{k}>|H| \text {. }
$$

By assumption $H_{0}=\sup |H|<1$. Using (19) it follows that (21) holds if we choose $C>0$ such that

$$
C>\sqrt{\frac{H_{0}^{2}}{1-H_{0}^{2}}} \geq \sqrt{\frac{H_{0}^{2}}{H_{k}^{2}-H_{0}^{2}}} \geq \sqrt{\frac{H^{2}}{H_{k}^{2}-H^{2}}} .
$$

We have for $x \in \Gamma_{k}$ that $v_{k}(x) \geq\left. F\right|_{\Gamma_{k}}(x)$. We conclude that $v_{k}$ is an upper barrier to $u_{k}$, that is, $u_{k}(x) \leq v_{k}(x), x \in \Omega_{k}$. This implies that the functions $u_{k}$ have a uniform height estimate since $v_{k}(x) \leq M=M_{0}+C \pi$, and proves the claim.

It follows from the above height estimate and the gradient estimate in Theorem 1 that for given $m \in \mathbb{N}$ the sequence $\left\{\left.u_{k}\right|_{\bar{\Omega}_{m}}\right\}_{k>2 m}$ has equibounded $C^{1}$ norm in $\bar{\Omega}_{m}$. To be able to apply Theorem 1 we have to translate the solutions by $-M$. By elliptic theory this sequence has equibounded $C^{2, \alpha}$ norm in $\Omega_{m}$. Then, there is a subsequence $\left\{u_{k_{j}^{m}}\right\}_{j}$ of $\left\{u_{k}\right\}_{k>2 m}$ converging on $\Omega_{m}$ in the $C^{2}$ norm to a function $v_{m} \in C^{2}\left(\Omega_{m}\right)$. Denoting $Q_{H}[u]=\mathcal{Q}[u]-n H$, we have that $Q_{H}\left[v_{m}\right]=0$. It follows from PDE regularity (cf. [7]) that $v_{m} \in C^{2, \alpha}\left(\Omega_{m}\right)$.

Consider a subsequence $\left\{u_{k_{j}^{m+1}}\right\}_{j}$ of $\left\{u_{k_{j}^{m}}\right\}_{j}$ which converges in $\Omega_{m+1}$ to a function $v_{m+1} \in C^{2, \alpha}\left(\Omega_{m+1}\right)$ satisfying $Q_{H}\left[v_{m+1}\right]=0$. After iterating this process, we obtain a 
subsequence $\left\{u_{k_{1+i}^{m+i}}\right\}_{i}$ of $\left\{u_{k}\right\}_{k}$ that converges uniformly on compact subsets of $\mathbb{H}^{n}$ in the $C^{2}$ norm to a function $u \in C^{2}\left(\mathbb{H}^{n}\right)$ satisfying $Q_{H}[u]=0$. By simplicity, we also denote this subsequence by $\left\{u_{k}\right\}_{k \in \mathbb{N}}$. We claim that $u$ extends continuously to $\partial_{\infty} \mathbb{H}^{n}$ and that $\left.u\right|_{\partial_{\infty} \mathbb{H}^{n}}=\phi$.

We first prove that $\partial_{\infty} \operatorname{Gr}(u) \subset \operatorname{Gr}(\phi)$ by showing that if $p \in \partial_{\infty} \mathbb{H}^{n+1} \backslash \operatorname{Gr}(\phi)$ then $p \notin \partial_{\infty} \operatorname{Gr}(u)$. Consider $p \in \partial_{\infty} \mathbb{H}^{n+1} \backslash \operatorname{Gr}(\phi)$. Since $\operatorname{Gr}(\phi)$ is compact and $p \notin \operatorname{Gr}(\phi)$ there exists an equidistant hypersurface $E$ of $\mathbb{H}^{n+1}$ such that $\partial_{\infty} E$ separates $p$ from $\operatorname{Gr}(\phi)$, that is, $p$ and $\operatorname{Gr}(\phi)$ are in distinct open connected components of $\partial_{\infty} \mathbb{H}^{n+1} \backslash \partial_{\infty} E$. Moreover, since $H_{0}=\sup _{\mathbb{H}^{n}}|H|<1$, we may assume that $E$ has constant mean curvature $H_{0}$ with respect to the unit normal vector field pointing to the connected component $U$ of $\mathbb{H}^{n+1} \backslash E$ whose asymptotic boundary contains $\operatorname{Gr}(\phi)$. Set $G_{k}=\operatorname{Gr}\left(u_{k}\right)$. By the convergence of $\left.u_{k}\right|_{\Gamma_{k}}$ to $\phi$ there is $k_{0}$ such that $\partial G_{k} \subset U$ and then $\partial G_{k} \cap E=\emptyset$ for all $k \geq k_{0}$. By the tangency principle $G_{k} \cap E=\emptyset$, i.e., $G_{k} \subset U$ for all $k \geq k_{0}$. It follows that $p \notin \partial_{\infty} \operatorname{Gr}(u)$.

Now consider a sequence $x_{k} \in \mathbb{H}^{n}$ converging to $x \in \partial_{\infty} \mathbb{H}^{n}$. By the compactness of $\overline{\mathbb{H}}^{n+1}$ there is a subsequence $\Psi\left(u\left(x_{k_{j}}\right), x_{k_{j}}\right)$ of $\Psi\left(u\left(x_{k}\right), x_{k}\right)$ converging to $z \in \overline{\mathbb{H}}^{n+1}$. Since $x_{k}$ diverges and $\Psi\left(u\left(x_{k_{j}}\right), x_{k_{j}}\right) \in \operatorname{Gr}(u)$ it follows that $z \in \partial_{\infty} \operatorname{Gr}(u)$. From what we proved above $z \in \operatorname{Gr}(\phi)$ and hence $z=\Psi\left(\phi\left(x_{0}\right), x_{0}\right)$ for some $x_{0} \in \partial_{\infty} \mathbb{H}^{n}$. Since $u$ is globally bounded, the sequence $\left\{u\left(x_{k_{j}}\right)\right\}_{j} \subset \mathbb{R}$ is bounded and thus contains a subsequence $\left\{u\left(x_{k_{j_{i}}}\right)\right\}_{i}$ converging to some $s_{0} \in \mathbb{R}$. Being the extension of $\Psi_{s_{0}}$ to $\overline{\mathbb{H}}^{n+1}$ continuous, we obtain that

$$
z=\lim _{i} \Psi\left(u\left(x_{k_{j_{i}}}\right), x_{k_{j_{i}}}\right)=\Psi\left(s_{0}, x\right)
$$

and therefore $\Psi\left(\phi\left(x_{0}\right), x_{0}\right)=\Psi\left(s_{0}, x\right)$. Since $\Psi: \mathbb{R} \times \partial_{\infty} \mathbb{H}^{n} \rightarrow \partial_{\infty} \mathbb{H}^{n+1}$ is injective, it follows that $x=x_{0}$ and $s_{0}=\phi\left(x_{0}\right)$. Because the limits are the same for any convergent subsequence considered, it follows that $u\left(x_{k}\right) \rightarrow \phi(x)$ as $k \rightarrow \infty$, and this proves the claim.

Finally, uniqueness follows from the maximum principle applied to the difference of two solutions.

\section{References}

[1] B. Guan and J. Spruck. Hypersurfaces of constant mean curvature in hyperbolic space with prescribed asymptotic boundary at infinity. Amer. J. Math. 122 (2000), 1039 1060 .

[2] M. Dajczer, P. Hinojosa and J. H. de Lira. Killing graphs with prescribed mean curvature. Calc. Var. Partial Differential equations 33 (2008), 231-248.

[3] K. Ecker and G. Huisken. Interior estimates for hypersurfaces moving by mean curvature. Invent. math. 105 (1991), 547-569. 
[4] M. Eichmair. The Plateau problem for marginally outer trapped surfaces. J. Diff. Geometry 83 (2009), 551-583.

[5] N. do Espírito-Santo, S. Fornari and J. Ripoll. The Dirichlet problem for the minimal hypersurface equation in $M \times \mathbb{R}$ with prescribed asymptotic boundary. J. Math. Pures Appl. 93 (2010), 204-221.

[6] J. Gálvez and H. Rosenberg. Minimal surfaces and harmonic diffeomorphisms from the complex plane onto certain Hadamard surfaces. Amer. J. of Math. 132 (2010), $1249-1273$.

[7] D. Gilbarg and N. Trudinger. Elliptic partial differential equations of second order. Springer Verlag, Berlin-Heidelberg, 2001.

[8] N. Korevaar. An easy proof of the interior gradient bound for solutions to the prescribed mean curvature problem. Proc. Symp. Pure Math. 45 (1986), 81-89.

[9] N. Korevaar. A priori interior gradient bounds for solutions to elliptic Weingarten equations. Ann. Inst. H. Poincaré Anal. Non Linéaire 4 (1987), 405-421.

[10] J. H. de Lira and G. Wanderley. Existence of nonparametric solutions for a capillary problem in warped products. http://arxiv.org/abs/1307.2871

[11] J. Ripoll and M. Telichevesky, On the asymptotic Dirichlet problem for some divergence form quasi-linear elliptic PDE's. To appear on the Transactions of the AMS.

[12] J. Serrin, Gradient estimates for solutions of nonlinear elliptic and parabolic equations. Contributions to nonlinear functional analysis (Proc. Sympos., Math. Res. Center, Univ. Wisconsin, Madison, Wis., 1971), 565-601. Academic Press, New York, 1971.

[13] J. Spruck. Interior gradient estimates and existence theorems for constant mean curvature graphs in $M \times \mathbb{R}$. Pure and Applied Mathematics Quarterly 3 (2007), 785-800.

Marcos Dajczer

IMPA

Estrada Dona Castorina, 110

22460-320 - Rio de Janeiro - Brazil

marcos@impa.br 
Jorge Herbert de Lira

Departamento de Matematica, Universidade Federal do Ceara, Bloco 914 - Campus do Pici 60455-760 - Fortaleza - Ceara - Brazil jorge.lira@mat.ufc.br

Jaime Ripoll

Departamento de Matematica Univ. Federal do Rio Grande do Sul Av. Bento Gonçalves 9500 91501-970 - Porto Alegre - RS - Brazil jaime.ripoll@ufrgs.br 